\title{
Percepção de estresse: associação com a prática de atividades físicas no lazer e comportamentos sedentários em trabalhadores da indústria
}

CDD. 20.ed. 155.9042 613.7

\author{
Breno Quintella FARAH* \\ Mauro Virgílio Gomes de BARROS* \\ José Cazuza de FARIAS JÚNIOR ${ }^{* *}$ \\ Raphael Mendes RITTI-DIAS* \\ Rodrigo Antunes LIMA* \\ João Paulo dos Anjos Souza BARBOSA* \\ Markus Vinicius NAHAS ${ }^{* * *}$ \\ *Universidade de
Pernambuco.
**Universidade Federal
da Paraiba.
***Universidade
Federal de Santa
Catarina.
}

\begin{abstract}
Resumo
0 objetivo deste estudo foi analisar a prevalência e a associação entre inatividade física no lazer e comportamento sedentário com a percepção negativa de estresse em industriários. Para tanto, 1910 trabalhadores foram selecionados mediante amostragem em dois estágios entre os industriários pernambucanos. Dados foram obtidos mediante uso de questionário previamente validado (Estilo de Vida e Hábitos de Lazer do Trabalhador da Indústria). Percepção negativa de estresse foi referida por 13,2\% e $45,2 \%$ dos trabalhadores eram fisicamente inativos no lazer. A proporção de sujeitos que relatou despender $>2$ horas/dia assistindo televisão foi de $31,8 \%$ e $57,4 \%$, respectivamente, em dias da semana e no final de semana. Observou-se, entre os homens, uma associação positiva entre o tempo de assistência à televisão em dias de semana e a percepção negativa de estresse. Conclui-se que a exposição a comportamento sedentário nos homens está associada à maior chance de percepção negativa de estresse.
\end{abstract}

Palavras-chave: Estresse psicológico; Atividade física; Sedentarismo; Trabalhadores.

\section{Introdução}

O estresse é uma condição que pode afetar pessoas de todas as idades, no entanto, alguns subgrupos populacionais parecem ser mais vulneráveis, como mulheres, idosos, pessoas mais pobres e indivíduos com baixo nível de escolaridade ${ }^{1}$. Os trabalhadores da indústria também constituem um subgrupo populacional exposto a agentes estressores específicos ${ }^{2-4}$.

Níveis elevados de estresse estão positivamente associados ao excesso de peso ${ }^{5}$, tabagismo ${ }^{6-7}$, hábitos alimentares inadequados ${ }^{8-9}$ e elevação dos níveis de pressão arterial ${ }^{10-11}$. Estima-se que o risco de um acidente vascular cerebral é o dobro em trabalhadores estressados ${ }^{12}$ e que $32,5 \%$ dos infartos do miocárdio são decorrentes de fatores psicossociais, dentre os quais o estresse e a ansiedade ${ }^{13}$.
Dentre as estratégias disponíveis para o controle do estresse, a prática de exercícios físicos vem sendo apontada como uma opção acessível e eficaz ${ }^{14}$. Ademais, estudos com trabalhadores revelaram que aqueles com maior nível de atividade física no lazer apresentavam uma menor percepção negativa de estresse $e^{2,6,15-17}$.

Uma abordagem de investigação que vem sendo proposta é analisar simultaneamente como a prática de atividades físicas e a exposição a comportamentos sedentários (exemplos: assistir à televisão, ler e usar o computador) podem estar associados a eventos de saúde ${ }^{18-22}$. Entretanto, em estudos com trabalhadores brasileiros, não se investigou se a exposição a comportamentos sedentários, independentemente do nível de atividade física, poderia ser um fator associado à percepção negativa de estresse. Tais 
informações possibilitariam a criação de estratégias para redução do nível de estresse nesse grupo populacional notoriamente exposto a fatores estressores. Além disso, até onde se tem conhecimento, poucos estudos foram realizados ${ }^{2-4}$ a fim de determinar a prevalência desses indicadores comportamentais de saúde em trabalhadores do setor industrial.

\section{Método}

\section{Caracterização do estudo}

Para desenvolvimento desse estudo transversal foi realizada uma análise secundária dos dados do projeto "Estilo de Vida e Hábitos de Lazer dos Trabalhadores da Indústria Brasileira”, desenvolvido pelo Serviço Social da Indústria (SESI) em parceria com o Núcleo de Pesquisa em Atividade Física e Saúde (NuPAF) da Universidade Federal de Santa Catarina. O protocolo de pesquisa foi aprovado pelo Comitê de Ética em Pesquisa com seres humanos da Universidade Federal de Santa Catarina (protocolo no 306/2005).

\section{População alvo e amostra}

O Estado de Pernambuco tinha, no ano de 2006, aproximadamente 133 mil trabalhadores atuando em 829 empresas, segundo dados fornecidos pelo SESI de Pernambuco, que possuía as melhores informações sobre quantitativo e distribuição dos trabalhadores da indústria no Estado, o que ajudou a subsidiar o planejamento amostral do estudo. Os participantes foram selecionados mediante amostragem em dois estágios, de modo a ser representativa do conjunto de indústrias do Estado. No primeiro estágio, a unidade amostral foi as empresas do setor industrial selecionadas por amostragem aleatória, estratificada segundo porte (grande, médio e pequeno). No segundo estágio, em cada uma das empresas sorteadas, foram selecionados, de forma sistemática, os trabalhadores.

O tamanho mínimo da amostra foi calculado considerando um erro amostral de três pontos percentuais, intervalo de confiança de $95 \%$, uma prevalência de inatividade física no lazer de $46 \%{ }^{2}$, acrescendo-se $50 \%$ a este número em função do delineamento amostral (efeito do delineamento amostral $=1,5$ ) e mais $20 \%$ para possíveis perdas no processo de coleta. Isto resultou numa amostra estimada em cerca de 1900 trabalhadores.
Buscando preencher essa lacuna de conhecimento, o objetivo deste estudo foi analisar a prevalência e a associação entre inatividade física no lazer e comportamento sedentário com a percepção negativa de estresse em industriários. Procurou-se também analisar as diferenças intersexuais quanto à prevalência e à associação destes indicadores de saúde.

\section{Coleta de dados}

Na coleta dos dados, utilizou-se o Questionário Estilo de Vida e Hábitos de Lazer dos Trabalhadores da Indústria, que permite obter dados demográficos e socioeconômicos, indicadores de saúde e bemestar, morbidade referida de doenças crônicas não transmissíveis, atividades físicas de lazer, participação em programas de ginástica na empresa, controle do peso corporal e hábitos alimentares. As características psicométricas deste instrumento, notadamente indicadores de validade de face e de reprodutibilidade, foram relatadas em estudo prévio $^{23}$. Questionários contendo as mesmas perguntas e as mesmas estratégias para definição operacional das variáveis foram utilizados em estudos prévios ${ }^{2-3}$.

Os dados foram coletados durante o primeiro semestre de 2006. A aplicação do questionário foi realizada em pequenos grupos de trabalhadores (três a 15 trabalhadores) por uma equipe previamente treinada visando à padronização dos procedimentos de coleta.

\section{Variáveis do estudo}

A percepção do estresse (variável dependente) foi mensurada a partir de uma única pergunta ("como você classifica o nível de estresse em sua vida?"), admitindo-se quatro opções de respostas: 1) raramente estressado; 2) as vezes estressado, vivendo razoavelmente bem; 3) quase sempre estressado; e, 4) sempre estressado, com dificuldade para enfrentar a vida diária. Foram classificados como casos de percepção negativa de estresse os sujeitos que referiram as respostas 3 ou 4 , a exemplo do que foi adotado em estudos congêneres ${ }^{2-3}$.

Para medida do nível de atividade física no lazer (variável independente), foi utilizada uma única pergunta: "você realiza, regularmente, algum tipo 
de atividade física no seu lazer, tais como exercícios físicos (ginástica, caminhada, corrida), esportes, ou artes marciais?”. As respostas possíveis eram: 1) não faz e não tem interesse; 2) não faz, mas tem interesse; 3) faz atividade física uma duas vezes por semana;

4) faz atividade física três a quatro vezes por semana; e, 5) faz atividade física cinco ou mais vezes por semana. Foram considerados fisicamente inativos no lazer os trabalhadores que relataram que não faziam atividade física no lazer (respostas 1 ou 2). Esta estratégia de classificação do nível de atividade física está em conformidade com o adotado em estudos similares ${ }^{3,24}$.

Foi utilizado como referência de comportamento sedentário (variável independente) o tempo de assistência à televisão. Esta opção metodológica foi decorrente do uso frequente deste tipo de medida em estudos epidemiológicos ${ }^{25}$, além de ser o principal indicador de comportamento sentado não relacionado ao trabalho ${ }^{26}$. Foram considerados expostos a elevado tempo de assistência à televisão aqueles que referiram assistir mais de duas horas diárias, optando-se por analisar essa variável separadamente, considerado a conduta adotada em dias de semana e nos dias de final de semana. Esta opção foi decorrente do delineamento metodológico observado em estudos anteriores ${ }^{20,22}$.

Também foram consideradas as seguintes potenciais variáveis de confusão: gênero (masculino; feminino); faixa etária ( $\leq 39$ anos; >39 anos); renda familiar bruta em reais $(\leq 1,5$ mil reais; $>1,5$ mil reais); estado civil (casado; outro estado civil); e nível de escolaridade (ensino médio incompleto; ensino médio completo ou superior).

\section{Resultados}

A amostra foi composta por 1910 sujeitos $(78,5 \%$ homens), em sua maioria $(56,4 \%)$ trabalhadores de empresas de grande porte. Mais da metade dos industriários tinham idade superior a 39 anos $(69,5 \%)$ e $64,2 \%$ deles eram casados ou

\section{Tabulação e análise dos dados}

As informações coletadas foram digitalizadas a partir da leitura ótica dos questionários pelo programa Sphynx (Sphynx Software Solutions Incorporation, Washington, Estados Unidos), a fim de evitar erros de digitação. Em seguida, os dados foram exportados para o programa SPSS (Statistical Package for the Social Sciences), versão 17, no qual as análises estatísticas foram realizadas.

As análises abrangeram a utilização de procedimento de estatística descritiva (distribuição de frequência) e medidas de associação bivariável (teste de Qui-quadrado de Pearson) e multivariável (regressão logística binária). O teste do Qui-quadrado foi utilizado para comparar as proporções de trabalhadores da indústria expostos à inatividade física no lazer, ao comportamento sedentário e à percepção negativa de estresse entre as categorias das variáveis sociodemográficas. Estas análises foram realizadas a fim de explorar possíveis fatores de confusão e para identificar a necessidade de ajustamento estatístico das análises.

A regressão logística binária foi utilizada para expressar o grau de associação entre as variáveis independentes (atividade física no lazer e comportamento sedentário) e a variável dependente (percepção de estresse), recorrendo-se ao ajustamento para possíveis fatores de confusão. Inicialmente, as análises foram ajustadas para fatores demográficos e socioeconômicos (ajustamento parcial) e, por fim, para as demais variáveis independentes consideradas no estudo (ajustada). Em todas as análises foi adotado um nível de significância de $\mathrm{p}<0,05$. Os valores $\mathrm{p}$, quando pertinente, foram apresentados no texto, figuras e tabelas.

viviam com parceiro(a). Mulheres apresentaram maior nível de escolarização e renda familiar bruta. $\mathrm{Na}$ TABELA 1 estão apresentadas as características demográficas e socioeconômicas dos participantes do estudo. 
TABELA 1 - Características demográficas dos trabalhadores da indústria do Estado de Pernambuco.

\begin{tabular}{|c|c|c|c|}
\hline Variável & Masculino n (\%) & Feminino n (\%) & Todos n (\%) \\
\hline \multicolumn{4}{|l|}{ Tamanho da empresa } \\
\hline Pequena & $261(17,4)$ & $128(31,1)$ & $389(20,4)$ \\
\hline Média & $393(26,2)$ & $105(25,6)$ & $498(26,0)$ \\
\hline Grande & $845(56,4)$ & $178(43,3)$ & $1023(53,6)$ \\
\hline \multicolumn{4}{|l|}{ Faixa etária } \\
\hline$<30$ anos & $501(33,4)$ & $175(42,6)$ & $676(35,4)$ \\
\hline 30-39 anos & $529(35,3)$ & $122(29,7)$ & $651(34,1)$ \\
\hline $40-49$ anos & $324(21,6)$ & $95(23,1)$ & $419(21,9)$ \\
\hline $50+$ anos & $145(9,7)$ & $19(4,6)$ & $164(8,6)$ \\
\hline \multicolumn{4}{|l|}{ Estado civil } \\
\hline Solteiro & $395(26,3)$ & $216(52,5)$ & $611(32,0)$ \\
\hline Casado ou vivendo com parceiro & $1064(71,0)$ & $162(39,4)$ & $1226(64,2)$ \\
\hline Viúvo & $9(0,6)$ & $4(1,0)$ & $13(0,7)$ \\
\hline Separado & $31(2,1)$ & $29(7,1)$ & $60(3,1)$ \\
\hline \multicolumn{4}{|l|}{ Nível de escolarizaçáo } \\
\hline Fundamental incompleto & $497(33,1)$ & $52(12,7)$ & $549(28,7)$ \\
\hline Fundamental completo & $232(15,5)$ & $51(12,4)$ & $283(14,8)$ \\
\hline Médio completo & $695(46,4)$ & $233(56,7)$ & $928(48,6)$ \\
\hline Superior completo & $75(5,0)$ & $75(18,2)$ & $150(7,9)$ \\
\hline \multicolumn{4}{|l|}{ Renda familiar bruta } \\
\hline Até 600 reais & $760(50,7)$ & $155(37,8)$ & $915(47,9)$ \\
\hline $600,01-1.500$ reais & $591(39,4)$ & $162(39,4)$ & $753(39,4)$ \\
\hline $1.500,01-3.000$ reais & $101(6,8)$ & $63(15,3)$ & $164(8,6)$ \\
\hline$>3.000,01$ reais & $47(3,1)$ & $31(7,5)$ & $78(4,1)$ \\
\hline
\end{tabular}

Verificou-se que $45,2 \%$ dos trabalhadores eram fisicamente inativos no lazer e 13,2\% referiram-se a uma percepção negativa de estresse. Além disso, $31,8 \%$ relataram assistir mais de 2 horas/dia de televisão em dias da semana, enquanto em dias do final de semana este percentual foi de $57,4 \%$. Nas mulheres, observou-se uma maior prevalência de inatividade física no lazer $(66,2 \%)$ e uma maior percepção negativa de estresse $(20,9 \%)$ em comparação ao observado entre os homens. Por sua vez, entre os homens, a exposição a comportamentos sedentários foi mais prevalente comparado às mulheres, conforme resultados apresentados na TABELA 2.

A prevalência de percepção negativa de estresse foi significativamente maior entre as mulheres e entre os trabalhadores fisicamente inativos no lazer (TABELA 3). Não foi observada diferença entre os trabalhadores com maior ou menor tempo de assistência à televisão quanto à prevalência de percepção negativa de estresse. 
TABELA 2 - Inatividade física no lazer, comportamento sedentário e percepção de estresse, estratificado por gênero.

\begin{tabular}{|c|c|c|c|c|}
\hline Variável & $\begin{array}{c}\text { Masculino } \\
\text { n (\%) }\end{array}$ & $\begin{array}{c}\text { Feminino } \\
\text { n (\%) }\end{array}$ & $\begin{array}{l}\text { Todos } \\
\text { n (\%) }\end{array}$ & $\mathbf{p}$ \\
\hline Inatividade física no lazer & & & & $<0,001$ \\
\hline Não & $908(60,6)$ & $139(33,8)$ & $1047(54,8)$ & \\
\hline Sim & $591(39,4)$ & $272(66,2)$ & $863(45,2)$ & \\
\hline Assistir TV (dias por semana) & & & & 0,026 \\
\hline Até 2 horas & $1004(67,0)$ & $299(72,7)$ & $1303(68,2)$ & \\
\hline Mais de 2 horas & $495(33,0)$ & $112(27,3)$ & $607(31,8)$ & \\
\hline Assistir TV (dias do fim de semana) & & & & 0,046 \\
\hline Até 2 horas & $621(41,5)$ & $193(47,0)$ & $814(42,6)$ & \\
\hline Mais de 2 horas & $877(58,5)$ & $218(53,0)$ & $1095(57,4)$ & \\
\hline Percepçáo de estresse & & & & $<0,001$ \\
\hline Positiva & $1333(88,9)$ & $325(79,1)$ & $1658(86,8)$ & \\
\hline Negativa & $166(11,1)$ & $86(20,9)$ & $252(13,2)$ & \\
\hline
\end{tabular}

TABELA 3 - Prevalência e fatores associados à percepção negativa de estresse.

\begin{tabular}{|c|c|c|c|}
\hline \multirow{2}{*}{ Variáveis } & \multicolumn{3}{|c|}{ Percepção negativa de estresse } \\
\hline & n (\%) & $\chi^{2}$ & $\mathbf{p}$ \\
\hline \multicolumn{4}{|l|}{ Gênero } \\
\hline Masculino & $166(11,1)$ & 27,328 & $<0,001$ \\
\hline Feminino & $86(20,9)$ & & \\
\hline \multicolumn{4}{|l|}{ Faixa etária } \\
\hline Até 39 anos & $171(12,9)$ & 0,359 & 0,549 \\
\hline Mais de 39 anos & $81(13,9)$ & & \\
\hline \multicolumn{4}{|l|}{ Estado civil } \\
\hline Casado & $167(13,6)$ & 0,547 & 0,460 \\
\hline Outros & $85(12,4)$ & & \\
\hline \multicolumn{4}{|l|}{ Nível de escolarizaçãoo } \\
\hline Até o fundamental completo & $106(12,7)$ & 0,256 & 0,607 \\
\hline Ensino médio ou mais & $146(13,5)$ & & \\
\hline \multicolumn{4}{|l|}{ Renda familiar bruta } \\
\hline Até 1500 reais & $213(12,8)$ & 2,066 & 0,151 \\
\hline Mais que 1500 reais & $39(16,1)$ & & \\
\hline \multicolumn{4}{|l|}{ Inatividade física no lazer } \\
\hline Não & $118(11,3)$ & 7,485 & 0,006 \\
\hline Sim & $134(15,5)$ & & \\
\hline \multicolumn{4}{|l|}{ Assistir TV (dias por semana) } \\
\hline Até 2 horas & $167(12,8)$ & 0,509 & 0,571 \\
\hline Mais de 2 horas & $85(14,0)$ & & \\
\hline \multicolumn{4}{|l|}{ Assistir TV (dias do fim de semana) } \\
\hline Até 2 horas & $111(13,6)$ & 0,235 & 0,519 \\
\hline Mais de 2 horas & $141(12,9)$ & & \\
\hline
\end{tabular}


Na TABELA 4 estão apresentadas as análises de regressão logística binária. Nos homens, verificouse, após ajuste para potenciais variáveis de confusão, que o tempo de assistência à televisão em dias de semana está associado de forma positiva à percepção negativa de estresse. Após ajuste para o nível de atividade física houve aumento na magnitude da medida de associação.

TABELA 4 - Análise de regressão logística (bruta, ajustado parcialmente e ajustado) das variáveis independentes estratificado pelo gênero.

\begin{tabular}{|c|c|c|c|c|c|c|c|}
\hline Variáveis & Categorias & $\begin{array}{c}\text { OR } \\
\text { bruta }\end{array}$ & IC95\% & $\begin{array}{c}\text { OR } \\
\text { parcialmente } \\
\text { ajustada }\end{array}$ & IC95\% & $\begin{array}{c}\text { OR } \\
\text { ajustada }\end{array}$ & IC95\% \\
\hline \multicolumn{8}{|l|}{ HOMENS } \\
\hline \multirow{2}{*}{ Inatividade física no lazer } & Não & 1 & & 1 & & 1 & \\
\hline & Sim & 1,20 & $0,86-1,67$ & 1,20 & $0,86-1,67$ & 1,20 & $0,86-1,68$ \\
\hline \multirow{2}{*}{ TV (fim de semana) } & $\leq 2 \mathrm{~h} / \mathrm{dia}$ & 1 & & 1 & & 1 & \\
\hline & $>2 \mathrm{~h} / \mathrm{dia}$ & 1,02 & $0,74-1,42$ & 1,01 & $0,72-1,40$ & 0,89 & $0,63-1,27$ \\
\hline \multirow{2}{*}{ TV (semana) } & $\leq 2 \mathrm{~h} / \mathrm{dia}$ & 1 & & 1 & & 1 & \\
\hline & $>2 \mathrm{~h} / \mathrm{dia}$ & 1,39 & $1,00-1,94$ & 1,39 & $0,99-1,93$ & $1,45^{*}$ & $1,01-2,06$ \\
\hline \multicolumn{8}{|l|}{ MULHERES } \\
\hline \multirow{2}{*}{ Inatividade física no lazer } & Não & 1 & & 1 & & 1 & \\
\hline & Sim & 1,42 & $0,84-2,39$ & 1,31 & $0,76-2,24$ & 1,30 & $0,75-2,23$ \\
\hline \multirow{2}{*}{ TV (fim de semana) } & $\leq 2 \mathrm{~h} / \mathrm{dia}$ & 1 & & 1 & & 1 & \\
\hline & $>2 \mathrm{~h} / \mathrm{dia}$ & 0,86 & $0,53-1,38$ & 0,91 & $0,56-1,47$ & 0,99 & $0,59-1,65$ \\
\hline \multirow{2}{*}{ TV (semana) } & $\leq 2 \mathrm{~h} / \mathrm{dia}$ & 1 & & 1 & & 1 & \\
\hline & $>2 \mathrm{~h} / \mathrm{dia}$ & 0,71 & $0,40-1,24$ & 0,75 & $0,42-1,32$ & 0,75 & $0,41-1,38$ \\
\hline
\end{tabular}

\section{Discussão}

Os principais achados relatados neste estudo foram: 1) as prevalências de inatividade física no lazer e de exposição a comportamento sedentário foram altas; 2) a prevalência de inatividade física no lazer foi maior entre as mulheres enquanto a exposição a comportamento sedentário foi mais prevalente entre os homens; e 3) a exposição a comportamento sedentário em homens está associada à percepção negativa de estresse.

A prevalência de inatividade física no lazer observada no presente estudo foi bastante inferior ao relatado em inquéritos realizados em Portugal $^{27} \mathrm{e}$ Colômbia ${ }^{28}$, onde as prevalências foram de 87,8\% e $79,1 \%$, respectivamente. No entanto, em investigaçōes realizadas em Santa Catarina ${ }^{2}$ e no Rio Grande do $\mathrm{Sul}^{24}$ com populaçóes de trabalhadores industriais, verificaram-se frequências semelhantes (46,2\% e 45,6\%, respectivamente). Assim, é possível supor que essas diferenças podem ser explicadas, em grande parte, pela delimitação mais abrangente da população alvo nos levantamentos internacionais em comparação ao realizado neste estudo, ao invés de fatores socioeconômicos e culturais.

A prevalência de inatividade física no lazer mais elevada entre as mulheres maior que a dos homens é um achado que converge para os resultados da ampla maioria dos estudos na área da epidemiologia da atividade física ${ }^{2-3,24,29}$. Pode-se especular que, nesta faixa etária, esta diferença intersexual decorre do maior envolvimento das mulheres em afazeres domésticos em adição à jornada de trabalho, fator que acaba reduzindo a disponibilidade de tempo para envolvimento em atividades físicas de lazer.

Similarmente ao observado em relação à inatividade física no lazer, a prevalência de exposição a comportamento sedentário observada no presente estudo foi inferior aos $69,5 \%$ verificados em estudo realizado na Escócia ${ }^{20}$. Essas diferenças podem ser atribuídas à maior idade dos sujeitos incluídos no estudo escocês 
(51 anos em média), tendo em vista que 91,4\% da amostra do presente estudo tinha idade inferior ou igual a 49 anos, e sabe-se, que adultos mais velhos apresentam maior exposição a comportamentos sedentários $^{29-30}$. Similarmente ao observado no presente estudo, outras investigações indicaram de modo consistente que a proporção de homens expostos ao comportamento sedentário é maior em comparação ao observado entre as mulheres ${ }^{20}$.

A prevalência de trabalhadores com percepção negativa de estresse está de acordo com resultados apresentados em outros estudos nacionais. Nesse sentido, BARros e NAHAS ${ }^{2}$ ao analisarem 4255 trabalhadores da indústria, observaram que 13,9\% tinham percepção negativa de estresse em sua vida. Da mesma forma, e utilizando o mesmo instrumento do presente estudo, FONSECA et al. ${ }^{3}$ observaram uma prevalência de 12,6\%. Em relação aos estudos internacionais, os resultados são controversos, uma vez que JONSDOTTIR et al. ${ }^{17}$ observaram 9\% de percepção negativa de estresse entre trabalhadores, CHANG et al. ${ }^{31}$ verificaram $24 \%$.

Tanto no presente estudo quanto em estudos anteriores ${ }^{2,32-33}$, observou-se uma maior prevalência de percepção negativa de estresse entre as mulheres em comparação aos homens. Uma possível explicação pode ser a jornada dupla de trabalho mais comum entre as mulheres ou a falta de acesso e de oportunidades às estratégias para controle de estresse, como a participação em programas estruturados de exercícios físicos ${ }^{14}$. No entanto, essa hipótese precisa ser testada em estudos subsequentes.

Diferentemente dos resultados relatados em estudos anteriores ${ }^{6,15-17,34}$ não foi verificada associação entre o nível de atividade física no lazer e a percepção de estresse. Diversos são os motivos que podem explicar esta discordância de resultados. Por exemplo, alguns estudos usaram escala de tensão no trabalho para mensurar o nível de estresse ${ }^{16}$, enquanto no presente estudo a medida foi derivada da percepção do trabalhador referida a partir de uma única questão. Outros estudos ${ }^{6,15,17,34}$ utilizaram como referência de atividade física no lazer a prática de exercícios físicos ou diferentes níveis de mensuração (sedentário, atividade física de baixa intensidade e alta intensidade) diferentemente da medida adotada no presente estudo.

Os resultados do presente estudo demonstraram existir uma associação direta entre o tempo de assistência à televisão em dias de semana e a percepção negativa de estresse, mas isto foi observado somente entre os homens. Este resultado está em consonância com investigação realizada com adultos escoceses ${ }^{20}$, na qual ficou demonstrada que os sujeitos que referiram assistir mais de duas horas de televisão por dia foram aqueles que apresentaram níveis mais elevados de estresse, independentemente do nível de atividade física no lazer. Todavia, não foram identificados outros estudos na literatura especializada que permitissem explorar comparativamente a associação entre essas variáveis. Ademais, os nexos de inter-relação entre estas variáveis precisarão ser esclarecidos mediante desenvolvimento de investigaçôes especificamente delineadas para este fim.

A plausibilidade para a associação entre comportamento sedentário (assistir televisão) e percepção negativa de estresse reside na hipótese de que o tempo excessivo gasto em frente à televisão possa acentuar o isolamento social e reduzir o envolvimento das pessoas em atividades com potencial para auxiliar no controle do estresse. De fato, há evidências de que o tempo de assistência à televisão está diretamente associado ao isolamento social e à limitação do desenvolvimento de redes de apoio social, fatores que podem afetar negativamente a saúde psicossocial ${ }^{35-36}$. Ademais, o excesso de tempo assistindo televisão, que geralmente ocorre antes de dormir, pode levar à interrupção do sono e ao aumento da disfunção cognitiva e mental ${ }^{37}$.

Até onde se tem conhecimento este é o primeiro estudo nacional com abrangência estadual que foi desenvolvido com a finalidade de analisar a prevalência e a associação entre a percepção de estresse com as práticas de atividades físicas no lazer e a exposição a comportamentos sedentários em trabalhadores industriais. Trata-se, portanto, de um estudo que poderá gerar novas ideias de pesquisa e auxiliar o desenvolvimento desta linha de investigação.

Em tempo, convém destacar que os leitores devem interpretar com cautela os resultados e as discussões relatadas neste artigo, pois há inúmeros aspectos que dificultam a comparabilidade dos resultados do presente estudo em relação a outras investigaçôes. Tais limitaçôes decorrem da diferente instrumentação adotada em cada estudo e, em certa medida, da escassez de estudos, focalizando simultaneamente as atividades físicas de lazer e o comportamento sedentário em industriários.

Além disso, deve-se destacar que o presente estudo tem outras limitações. Por exemplo, o uso de medidas autorreferidas possibilita a ocorrência de viés de informação, pois os trabalhadores expostos a maiores níveis de estresse podem ter uma capacidade diferenciada de recordar as atividades físicas praticadas e o tempo despendido assistindo televisão. 
Procurou-se atenuar este problema mediante utilização de um instrumento previamente testado e que apresentou bons índices de reprodutibilidade. Por ser um estudo de delineamento transversal, no qual as exposições (atividades físicas e comportamentos sedentários) e desfecho (percepção negativa de estresse) foram avaliados no mesmo período de tempo, há possibilidade de viés de causalidade reversa. Mas há também pontos positivos que devem ser destacados, uma vez que se trata de estudo conduzido com amostra relativamente grande e suficientemente precisa para as análises estatísticas propostas. Foi empregado procedimento probabilístico na seleção dos participantes, atenuando de certo modo a possibilidade de viés de seleção.

Os resultados deste estudo permitem concluir que as prevalências de inatividade física no lazer e comportamento sedentário são mais baixas do que o observado na população adulta em geral, mas são similares ao observado em outros estudos com trabalhadores industriais de outras regiōes do país. Além disso, a prevalência de inatividade física no lazer e de percepção negativa de estresse foi maior entre as mulheres, enquanto entre os homens foi observada maior prevalência de exposição a comportamento sedentário. Estas diferenças intersexuais definiram um perfil bidimensional: nos homens, os comportamentos de risco mais prevalentes tomam a forma de risco direto ou ativo (excesso de tempo assistindo televisão) enquanto entre as mulheres assumem a forma de risco indireto ou passivo (inatividade física no lazer, percepção negativa de estresse). Concluiu-se ainda que, entre os homens trabalhadores, a exposição a comportamento sedentário é fator de risco para percepção negativa de estresse.

A partir destes achados e considerando as suas implicações sanitárias, sugere-se que as campanhas e intervenções que focalizem a redução do estresse possam considerar a possibilidade de intervenção na redução de tempo de exposição a comportamentos sedentários. É preciso também desenvolver mais estudos a fim de esclarecer os mecanismos que podem explicar esta associação.

\begin{abstract}
Perceived stress: association with the practice of leisure-time physical activity and sedentary behaviors in industrial workers

The aim of this study was to analyze the prevalence and the association between physical activity and sedentary behaviors with higher perceived stress in industrial workers. For this purpose, we used a crosssectional design in which participants were selected using a 2-stage sampling process among industrial workers in Pernambuco state (northeast of Brazil). Data were obtained by using a previously validated questionnaire (Lifestyle and Leisure Habits of Industrial Workers). Higher perceived stress was referred by $13.2 \%$ and $45.2 \%$ were inactive during leisure-time. The prevalence of workers who reported spending $>2$ hours/day watching television was $31.8 \%$ and $57.4 \%$, respectively, in week and in weekend days. It was observed, among men, a positive association between the TV-watching hours in week days with a higher perceived stress. It was concluded that the likelihood of referring higher perceived stress was greater among men exposed to sedentary behavior.
\end{abstract}

KEY WORDS: Stress psychological; Motor activity; Sedentary lifestyle; Workers.

\title{
Referências
}

1. Sparrenberger F, Santos I, Lima RC. Epidemiology of psychological distress: a population-based cross-sectional study. Rev Saúde Pública. 2003;37:434-9.

2. Barros MVG, Nahas MV. Health risk behaviors, health status self-assessment and stress perception among industrial workers. Rev Saúde Pública. 2001;35:554-63.

3. Fonseca SA, Blank VL, Barros MV, Nahas MV. Self-perceived health and associated factors in industrial workers from Santa Catarina State, Brazil. Cad Saúde Pública. 2008;24:567-76. 
4. Silva SG, Silva MC, Nahas MV, Viana SL. Variables associated with leisure-time physical inactivity and main barriers to exercise among industrial workers in Southern Brazil. Cad Saude Pública. 2011:27:249-59.

5. Kouvonen A, Kivimaki M, Cox SJ, Cox T, Vahtera J. Relationship between work stress and body mass index among 45,810 female and male employees. Psychosom Med. 2005;67:577-83.

6. Ng DM, Jeffery RW. Relationships between perceived stress and health behaviors in a sample of working adults. Health Psychol. 2003;22:638-42.

7. Kouvonen A, Kivimaki M, Virtanen M, Pentti J, Vahtera J. Work stress, smoking status, and smoking intensity: an observational study of 46,190 employees. J Epidemiol Community Health. 2005;59:63-9.

8. Sims R, Gordon S, Garcia W, et al. Perceived stress and eating behaviors in a community-based sample of African Americans. Eat Behav. 2008;9:137-42.

9. Nishitani N, Sakakibara H, Akiyama I. Eating behavior related to obesity and job stress in male Japanese workers. Nutrition. 2009;25:45-50.

10. Guimont C, Brisson C, Dagenais GR, et al. Effects of job strain on blood pressure: a prospective study of male and female white-collar workers. Am J Public Health. 2006;96:1436-43.

11. Gasperin D, Netuveli G, Dias-Da-Costa JS, Pattussi MP. Effect of psychological stress on blood pressure increase: a meta-analysis of cohort studies. Cad Saúde Pública. 2009;25:715-26.

12. Tsutsumi A, Kayaba K, Kario K, Ishikawa S. Prospective study on occupational stress and risk of stroke. Arch Intern Med. 2009;169:56-61.

13. Yusuf S, Hawken S, Ounpuu S, et al. Effect of potentially modifiable risk factors associated with myocardial infarction in 52 countries (the INTERHEART study): case-control study. Lancet. 2004;364:937-52.

14. He SB, Tang WG, Tang WJ, Kao XL, Zhang CG, Wong XT. Exercise intervention may prevent depression. Int J Sports Med. 2012;3:525-30.

15. Iwasaki Y, Zuzanek J, Mannell RC. The effects of physically active leisure on stress-health relationships. Can J Public Health. 2001;92:214-8.

16. Kouvonen A, Kivimaki M, Elovainio M, Virtanen M, Linna A, Vahtera J. Job strain and leisure-time physical activity in female and male public sector employees. Prev Med. 2005;41:532-9.

17. Jonsdottir IH, Rodjer L, Hadzibajramovic E, Borjesson M, Ahlborg GJ. A prospective study of leisure-time physical activity and mental health in Swedish health care workers and social insurance officers. Prev Med. 2010;51:373-7.

18. Rosenberg DE, Bull FC, Marshall AL, Sallis JF, Bauman AE. Assessment of sedentary behavior with the International Physical Activity Questionnaire. J Phys Act Health. 2008;5 Suppl 1:S30-44.

19. Martinez-Gomez D, Tucker J, Heelan KA, Welk GJ, Eisenmann JC. Associations between sedentary behavior and blood pressure in young children. Arch Pediatr Adolesc Med. 2009;163:724-30.

20. Hamer M, Stamatakis E, Mishra GD. Television- and screen-based activity and mental well-being in adults. Am J Prev Med. 2010;38:375-80.

21. Sisson SB, Broyles ST, Newton RL Jr, Baker BL, Chernausek SD. TVs in the bedrooms of children: does it impact health and behavior? Prev Med. 2011;52:104-8.

22. Stamatakis E, Hamer M, Dunstan DW. Screen-based entertainment time, all-cause mortality, and cardiovascular events: population-based study with ongoing mortality and hospital events follow-up. J Am Coll Cardiol. 2011;57:292-9.

23. Barros MVG. Atividades físicas no lazer e outros comportamentos relacionados à saúde dos trabalhadores da indústria no Estado de Santa Catarina, Brasil [dissertação]. Florianópolis (SC): Universidade Federal de Santa Catarina, Centro de Desportos; 1999.

24. Del Duca GF, Oliveira ESAD, Sousa TFD, Silva KSD, Nahas MV. Inatividade física no lazer em trabalhadores da indústria do Rio Grande do Sul, Brasil. Motriz: Rev Educ Fís. 2011;17:180-8.

25. Grontved A, Hu FB. Television viewing and risk of type 2 diabetes, cardiovascular disease, and all-cause mortality: a meta-analysis. JAMA. 2011;305:2448-55.

26. Clark BK, Sugiyama T, Healy GN, Salmon J, Dunstan DW, Owen N. Validity and reliability of measures of television viewing time and other non-occupational sedentary behaviour of adults: a review. Obes Rev. 2009;10:7-16.

27. Camoes M, Lopes C. Factors associated with physical activity in the Portuguese population. Rev Saúde Pública. 2008;42:208-16.

28. Gomez LF, Mateus JC, Cabrera G. Leisure-time physical activity among women in a neighbourhood in Bogota, Colombia: prevalence and socio-demographic correlates. Cad Saúde Pública. 2004;20:1103-9.

29. Hallal PC, Victora CG, Wells JC, Lima RC. Physical inactivity: prevalence and associated variables in Brazilian adults. Med Sci Sports Exerc. 2003;35:1894-900. 
Farah BQ, et al.

30. Martinez-Gonzalez MA, Varo JJ, Santos JL, et al. Prevalence of physical activity during leisure time in the European Union. Med Sci Sports Exerc. 2001;33:1142-6.

31. Chang SJ, Koh SB, Kang MG, et al. Epidemiology of psychosocial distress in Korean employees. J Prev Med Public Health. 2005;38:25-37.

32. Clays E, De Bacquer D, Leynen F, Kornitzer M, Kittel F, De Backer G. Job stress and depression symptoms in middleaged workers-prospective results from the Belstress study. Scand J Work Environ Health. 2007;33:252-9.

33. Hwang WJ, Hong O. Work-related cardiovascular disease risk factors using a socioecological approach: implications for practice and research. Eur J Cardiovasc Nurs. 2012;11:114-26.

34. Lutz RS, Lochbaum MR, Lanning B, Stinson LG, Brewer R. Cross-lagged relationships among leisure-time exercise and perceived stress in blue-collar workers. J Sport Exerc Psychol. 2007;29:687-705.

35. Wang JL. Perceived work stress, imbalance between work and family/personal lives, and mental disorders. Soc Psychiatry Psychiatr Epidemiol. 2006;41:541-8.

36. Rusli BN, Edimansyah BA, Naing L. Working conditions, self-perceived stress, anxiety, depression and quality of life: a structural equation modelling approach. BMC Public Health. 2008;8:48.

37. Dworak M, Schierl T, Bruns T, Struder HK. Impact of singular excessive computer game and television exposure on sleep patterns and memory performance of school-aged children. Pediatrics. 2007;120:978-85.

\section{Agradecimentos}

Ao Serviço Social da Indústria (SESI) pelo apoio logístico e institucional para a realização do estudo Perfil do Estilo de Vida e Hábitos de Lazer dos Industriários (Pernambuco - PE).

Ao Núcleo de Pesquisa em Atividade Física e Saúde da Universidade Federal de Santa Catarina pelo acesso ao banco de dados para desenvolvimento do estudo.

\begin{tabular}{r|l} 
ENDEREço & \\
Mauro Virgílio Gomes de Barros & \\
Universidade de Pernambuco & Recebido para publicação: 28/03/2012 \\
R. Arnóbio Marques, 310 & Revisado: 24/o9/2012 \\
50100-130 - Recife - PE - BRASIL & Aceito: 24/09/2012 \\
e-mail: maurovgb@gmail.com & \\
&
\end{tabular}

234 • Rev Bras Educ Fís Esporte, (São Paulo) 2013 Abr-Jun; 27(2):225-34 